\title{
VITASENIOR-MT: a telehealth solution for the elderly focused on the interaction with TV
}

\author{
Gabriel Pires, \\ VITA.IPT Lab. \\ Polytechnic Institute of Tomar \\ Tomar, Portugal \\ gppires@ipt.pt \\ Diogo Mendes, \\ VITA.IPT Lab. \\ Polytechnic Institute of Tomar \\ Tomar, Portugal \\ diogo.santos.mendes@ipt.pt \\ Pedro Ferreira \\ VITA.IPT Lab. \\ Polytechnic Institute of Tomar \\ Tomar, Portugal \\ pnlferreira@gmail.com \\ Luis Almeida \\ VITA.IPT Lab. \\ Polytechnic Institute of Tomar \\ Tomar, Portugal \\ laa@ipt.pt \\ Paulo Monteiro \\ Softinsa - Centro de Inovação \\ Tecnológica de Tomar, \\ Tomar, Portugal \\ paulo.monteiro@pt.softinsa.com
}

\author{
Pedro Correia \\ VITA.IPT Lab. \\ Polytechnic Institute of Tomar \\ Tomar, Portugal \\ pcorreia@ipt.pt \\ Nelson Gomes \\ VITA.IPT Lab. \\ Polytechnic Institute of Tomar \\ Tomar, Portugal \\ nelsonmpg@gmail.com \\ Ana Lopes \\ VITA.IPT Lab. \\ Polytechnic Institute of Tomar \\ Tomar, Portugal \\ anacris@ipt.pt \\ Luis Oliveira \\ VITA.IPT Lab. \\ Polytechnic Institute of Tomar \\ Tomar, Portugal \\ loliveira@ipt.pt \\ Carla Grácio \\ Comunidade Intermunicipal do Médio \\ Tejo (CIMT) \\ Tomar, Portugal \\ carla.gracio@cimt.pt
}

\author{
Dário Jorge \\ VITA.IPT Lab \\ Polytechnic Institute of Tomar \\ Tomar, Portugal \\ jorge.dario@ipt.pt \\ Pedro Dias \\ VITA.IPT Lab. \\ Polytechnic Institute of Tomar \\ Tomar, Portugal \\ diaspedro@ipt.pt \\ António Manso \\ VITA.IPT Lab. \\ Polytechnic Institute of Tomar \\ Tomar, Portugal \\ manso@ipt.pt \\ Renato Panda \\ VITA.IPT Lab. \\ Polytechnic Institute of Tomar \\ Tomar, Portugal \\ renato.panda@ipt.pt \\ Telmo Pereira \\ Escola Superior de Tecnologia da \\ Saúde de Coimbra do Instituto \\ Politécnico de Coimbra, \\ Coimbra, Portugal \\ telmo@estescoimbra.pt
}

\begin{abstract}
Remote monitoring of health parameters is a promising approach to improve the health condition and quality of life of particular groups of the population, which can also alleviate the current expenditure and demands of healthcare systems. The elderly, usually affected by chronic comorbidities, are a specific group of the population that can strongly benefit from telehealth technologies, allowing them to reach a more independent life, by living longer in their own homes. Usability of telehealth technologies and their acceptance by end-users are essential requirements for the success of telehealth implementation. Older people are resistant to new technologies or have difficulty in using them due to vision, hearing, sensory and cognition impairments. In this paper, we describe the implementation of an IoT-based telehealth solution designed specifically to address the elderly needs. The end-user interacts with a TV-set to record biometric parameters, and to receive warning and recommendations related to health and environmental sensor recordings. The familiarization of older people with the TV is expected to provide a more user-friendly interaction ensuring the effectiveness integration of the end-user in the overall telehealth solution.
\end{abstract}

Keywords-Telehealth, Elderly, Healthcare, TV interaction, Biometric data, Environmental data, Internet of Things (IoT).

\section{INTRODUCTION}

According to the European Commission projections, the continuous low birth rates and higher life expectancy will lead to a significant change of the EU-28 age population pyramid [1]. In 2060, it is expected that the elderly (aged 65 or more) account for $29.5 \%$ of the total population, and $12 \%$ of the total population will be aged 80 or more. The share of the working age population (between 15 and 64 years old) is expected to change from $67 \%$ in 2010 to $56.2 \%$ in 2060 [1]. The increased ageing and life expectancy have a direct consequence on the increased prevalence of chronic diseases, such as arterial hypertension, diabetes and dyslipidemia, thus contributing to the occurrence of major cardio-cerebrovascular events as the main causes of mortality and disability in almost all European Union countries [2]. This demographic and ageing change is leading to an increased demand of healthcare systems and to a transformation of the social, economic and healthcare models, which has been a main concern in many countries of the world in the last two decades. The elderly are one of the most vulnerable groups affected by this scenario, because of the increased comorbidity on one hand and the increased lack of caregivers on the other hand. Telehealth is a promising approach that can alleviate the expenditure of healthcare systems, and allow the elderly population to live independently in their own homes for longer periods of time. Telehealth may include services such as remote monitoring of biometric and environmental parameters, teleconsultation, monitoring of daily activity, communication, activities for daily living (physical exercise, entertainment), assistive mobility, rehabilitation, medication (see reviews in [3] [4] [5]). The benefits of remote health monitoring have been clearly shown, however, there are many issues still limiting its effective deployment. From a functionality and usability perspective, the proposed technologies are not always well accepted by end-users, hindering its successful deployment. Older people are quite resistant to new technologies or have difficulty in using them due to vision, hearing, sensory, dexterity and cognition impairments, which may render 
unfeasible the interaction with smartphones, tablets, or computers [4] [6]. Other interaction technologies have been considered in telehealth, for example, the use of the TV for user-system interaction has been shown to be a well-accepted approach by the elderly, as it gives them a sense of familiarity and confidence [7] [3]. Moreover, the TV offers other advantageous features, namely, almost everyone has one, the elderly are used to control their basic functions, the screen is large allowing to show large text and icons, and it is very easy to adjust the audio-level. Furthermore, the TV is always there in front of the sofa.

In this paper, we describe a telehealth solution under development called VITASENIOR-MT, specifically designed for monitoring health parameters of elderly people. Using an IoT architecture, this solution performs remote monitoring of physiological parameters of the elderly, as well as environmental parameters of their homes. The physiological variables include blood pressure, glucose, body weight and temperature measured on a daily basis by the senior's own initiative, but automatically recorded, and heart rate measurements collected automatically throughout the day, by means of a bracelet. Environmental parameters include temperature, humidity, carbon monoxide and carbon dioxide measurements. The TV occupies a featured position in this solution, it is used as the form of interaction between the enduser and the medical devices, as well as to receive medical recommendations, warnings and environmental alerts. It is expected that this device, very familiar to the elderly, will increase the acceptance and ease of use of the system. The solution is independent of the TV receiver and telecommunication operator, and was designed to minimize the required actions from the end-user. This paper focuses mainly on the implementation and functionality of the userTV interaction, but the overall architecture is also described.

VITASENIOR-MT project is being developed in a close collaboration with the Inter-municipal Community of the Medio Tejo region in Portugal. This region has a rate of ageing and population decrease higher than the national average. In some municipalities, the index of dependence is $70 \%$ and the population density is around 18 inhabitants $/ \mathrm{Km}^{2}$. In face of this scenario, municipalities of Medio Tejo carry out a continuous home support to provide assistance to the elderly population. Telehealth solutions are welcomed and seen as an important complement to domiciliary assistance.

\section{RELATED WORK}

Telehealth offers the possibility of monitoring the health parameters of people in their homes for short or long periods of time [5]. It can be used to help in medical diagnosis, in medical surveillance of patients during subacute periods, or for continuous monitoring of the population with chronic diseases, usually elderly or disabled, who do not need immediate medical care, but whose biometrical monitoring is important in accessing the evolution of health condition. Additionally, sensor networks are used for environmental monitoring or for tracking daily life activities, generating alarms in case of anomalous occurrences.

\section{A. System Architectures}

Telehealth monitoring architectures are composed of three main modules: biometric and ambient sensor networks, gateway, and cloud data storage and processing. Biometric sensors/devices can be connected through Bluetooth, WiFi or wire to a concentrator, or directly connected to a remote system through $3 \mathrm{G} / 4 \mathrm{G}$ networks. In more advanced systems, biometric sensors can be connected as wireless body area networks (WBAN), yet current implementations still suffer from several issues concerning wearability, power efficiency, cost, and hardware complexity [8]. Biometric signals/parameters may include EEG, ECG, blood pressure, heart rate, oximetry, glucose and others. Environmental monitoring and context analysis use wireless personal area networks, and include air quality sensors, humidity, video cameras, audio sensors, light sensors and others. The gateway, can be a general purpose device such as smartphone or tablet or a dedicated module [9]. Usually, each sensor has the ability to automatically connect to the gateway, which is responsible by its management in terms of sensor connectivity, authentication and security. The cloud data storage and processing module is responsible of managing the overall information, user access, data analysis and handling, generating reports to be used for the medical services and caregivers and sending alerts and useful reminders to the endusers [10] [5].

\section{B. User interaction}

With regard to user interaction in telehealth systems, many proposals are more focused on monitoring applications for medical staff and caregivers than for the end-users. For instance, authors in [11] propose a health service platform for elderly people, to detect dangerous events or anomalous behaviors, but the end-users are not included in the interaction loop, which is disadvantageous for user's acceptance and motivation. On the other hand, user interaction systems are often based on smartphones and tablets [12], but may also include interaction with service robots [13]. Although technologically interesting, these approaches are sometimes difficult to implement in real-life conditions given the profile of the senior population, and therefore other options should be considered, even if they are not as technologically advanced. For example, elderly users show a high degree of satisfaction towards the interaction with the TV set [14] [7]. In [15], a wireless health monitoring system is proposed, where users interact with a TV set to access biometric data. The connectivity between the medical devices and the gateway is done automatically. The system is based on a terrestrial digital video broadcasting - multimedia home platform (DVBTMHP) connected to the TV where the user can access and visualize historical data. However, this solution can only be implemented in countries that have MHP and DVBT return channel services available. Some commercial solutions based on TV interaction are also already available [16] [17], offering social interaction, like photo sharing and video conferencing, teleconsultation and health reminders.

\section{GLOBAL ARCHITECTURE OF VITASENIOR-MT}

Figure 1 shows the global architecture of VITASENIORMT telehealth solution and the actors interacting with it. The proposed solution follows an IoT paradigm that mainly consists of using embedded processors, sensors and communication hardware to collect, send and act on data they acquire from direct end-users and the environment. Collected data is then shared by connecting to an IoT gateway, the VITABOX, where data is either sent to the cloud to be stored and processed, or analyzed locally. VITASENIOR-MT comprises 4 main modules as described below. 


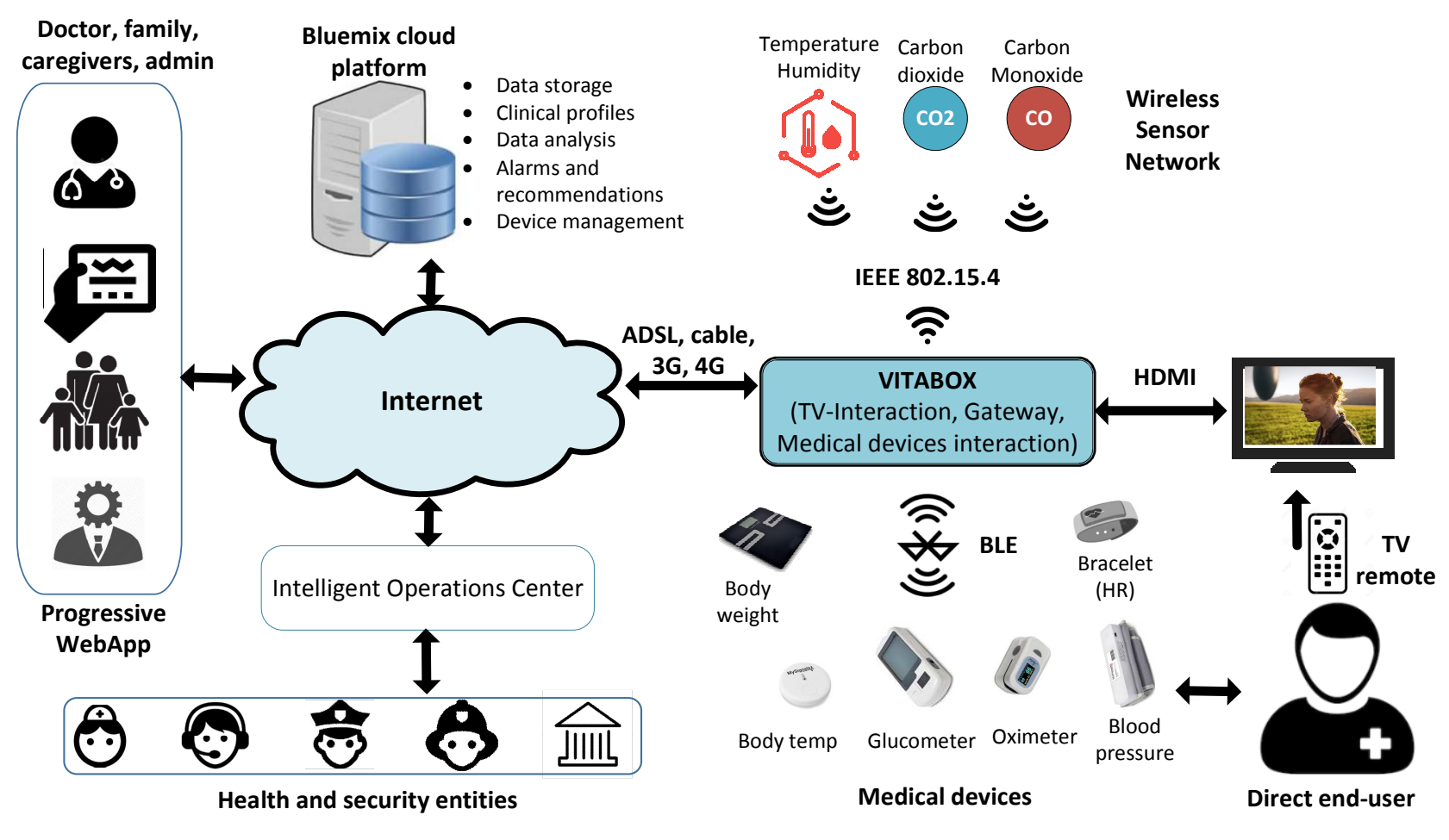

Fig. 1. Global architecture of VITASENIOR-MT.

\section{A. VITABOX}

VITABOX is the central component of the proposed solution: it manages the interaction between the end-user and the telehealth system though a TV set, it collects the user's physiological data recorded from the medical devices and assists the user in this procedure, it collects automatically biometric data from a wearable bracelet, and it provides a set of predefined recommendations and warnings to the end-user. Additionally, the VITABOX is the gateway of the residential network, sending all the environmental data and user's biometric data to the cloud. Section IV describes the full functionality of the VITABOX and its hardware and software architecture.

\section{B. Wireless sensor network: environmental sensors}

The wireless sensor network (WSN) is responsible for collecting environmental data from several rooms of the house. The WSN is composed by several nodes, equipped with transducers to monitor temperature, humidity, carbon monoxide $(\mathrm{CO})$ and carbon dioxide $\left(\mathrm{CO}_{2}\right)$ and by the border router. The border router is responsible to retrieve the data collected by the sensor nodes and to send them to the other systems when requested, periodically or when a predefined alarm occurs. Autonomous and resource constrained hardware devices are used in WSN in order to reduce the price of the solution and to extend its life time. The WSN solution is based on standard protocols such as IEEE802.15.4, 6LoWPAN and CoAP. The RPL protocol is used to establish a route-over mesh network, providing resilience and scalability to the WSN. The RPL and the 6LoWPAN messages exchanged between the WSN nodes during the normal operation are used to provide visibility about the connected nodes and the network topology, improving the management operations without increasing the overhead. The IEEE802.15.4 operating in AES-CCM mode provides four basic security services: access control, message integrity, message confidentiality, and replay protection. All nodes are configured in the provisioning phase with a pre-shared key and therefore only the nodes with the right key will join the network. Beyond this mechanism all nodes must be administratively approved by the network manager in order to provide more security to the network and to improve its manageability. The Zolertia Remote platform running Contiki operating system is in use in all WSN nodes.

\section{Cloud platform}

The users' data, including health and environmental information gathered by the VITABOXes, is stored in the VITASENIOR cloud infrastructure, hosted in the IBM cloud services. In addition to gathering data, the system allows different actors, such as the VITABOX users, their family members or health professionals to access it and obtain insights tailored to their needs and level of access. As an example, the user may access his historical data in a simplified view, while allowing a family member to see a more limited set of it. Health professionals using the system can access a health overview of their patients, as well as important episodes and detailed historical data. The live and historical users' data is also processed, generating alarms and recommendations when unexpected situations are identified. This information system follows a micro-services' approach, where a set of smaller services coexist, each responsible for a specific task. As a result of this approach, it is possible to isolate the data warehousing environment, from the service processing the background tasks' queue, the Application Programming Interface (API) nodes and the data analytics service. These are accessed through the Representational State Transfer (REST) API, based on NodeJS server environment, which uses the Google V8 engine due to its performance and scalability capabilities. Moreover, the cloud infrastructure supports the remote management of each VITABOX and associated 


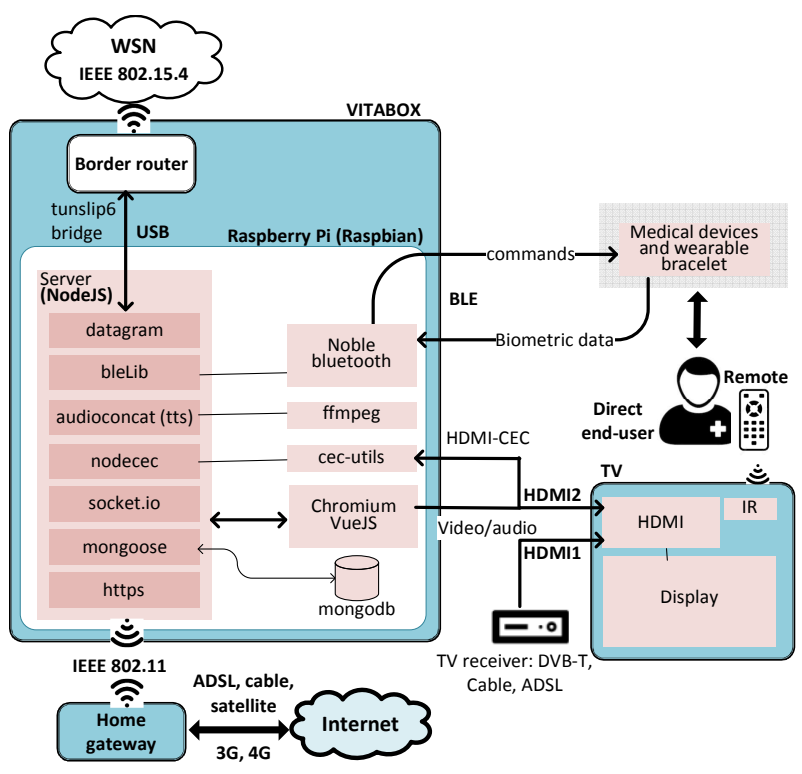

Fig. 2 VITABOX hardware and software architecture.

devices in its domain, the forwarding of complex data processing tasks to background workers through an Advanced Message Queueing Protocol (AMQP) server and the connection between the VITABOXes and the data hosting service. Secure communication between cloud infrastructure and clients is guaranteed through HTTP Secure (HTTPS), providing both authentication of the infrastructure, and privacy and integrity of the communications. The users' authentication system and session information is implemented using JSON Web Tokens (JWT) and the users' identification data stored in the cloud is encrypted. The users' frontend and administration back office are developed using VueJS framework, allowing a better user experience and at the same time lowering the resources needed in the server side.

\section{Intelligent Operations Center}

The intelligent operation center is the information system used to present and to alert the healthcare and security institutions about the events such as alarms and recommendations reported by the software layer located in the cloud. The reported events are presented in georeferenced web interface and associated with the event a list of procedures and the associated workflow is presented. Hence, this component not only identifies in the map where the event was occurred but also provides a list of the accepted procedures to accomplish the best response to the event. All the actions will be recorded in order to make easier key performance indicators determination and to audit the quality of the response procedures. This component is already specified and under development in the current project state.

\section{VITABOX}

\section{A. Hardware implementation}

The VITABOX is the central component of the entire architecture and the most critical one, because all the information passes through it. Moreover, it manages the interaction of the end-user with the overall system, which is the key point of this telehealth proposal. The VITABOX hardware and software architecture is depicted in Fig. 2.
VITABOX is based on a Raspberry Pi 3 (Rpi3) microcomputer running the Raspbian operating system. The Rpi3 integrates $2.4 \mathrm{GHz}$ WLAN IEEE $802.11 \mathrm{~b} / \mathrm{g} / \mathrm{n}$ and Bluetooth 4.1. It supports HDMI-Consumer Electronic Control (CEC), which allows a software-based way of programming the bidirectional interaction with the TV without requiring any additional external hardware. Using the standard infrared (IR) remote controller of the TV, it is possible to interact with the Rpi3 in a transparent way through HDMI-CEC commands. These features make RPi3 a suitable hardware solution fulfilling all our communication requisites. The TV set has to support HDMI-CEC and must have at least 2 HDMI ports, one for the VITABOX and the other for the set-top box (TV-receiver). These characteristics are present in almost all low-cost TV sets. The set-top box can be any one (DVB-T, DVB-S of public or private operators) and connected with any external source (terrestrial, satellite, cable, ADSL). An Internet access (home gateway) is required to deliver data to the cloud, receive warnings and recommendations, and enable external management of the home devices. In the rural areas, the Internet is typically provided over $3 \mathrm{G} / 4 \mathrm{G}$ or satellite networks. The border router of the WSN is connected to the RPi3 through an USB port using a serial line interface protocol (SLIP). The interaction between the VITABOX and all medical devices is made using Bluetooth low energy (BLE).

\section{B. Functionality and Telehealth-End-user Interaction}

The interaction of the end-user with the telehealth system is focused on the TV. The system provides several functionalities and operation modes, namely:

1) Alarm events: The alarm events are triggered when abnormal or irregular situations occur. These events could be related to concerning values of environmental data (e.g., carbon monoxide) or concerning values of physiological data of the end-user (e.g. blood pressure). When these events occur, the TV screen is automatically switched from HDMI1 to HDMI2, showing the alarm situation (as an example, see screenshot of Fig. 3c)). These events are also transmitted to the cloud platform, reaching predefined caregivers and the health and security entities through the IOC procedures (this last one, still under implementation). If the user is watching $\mathrm{TV}$, he/she will receive the alarm and its cause. The alarm will remain until the end-user presses the OK button of the TV remote control.

2) Notifications: notifications are recommendations and warnings generated in the cloud server, resulting from the analysis of historical data and taking previously inserted generic health protocols and user's specific clinical profiles. Some types of recommendations are: "Prolonged exposure to a given environmental parameter", "Days without measuring a given physiological parameter", "Prescription available", "Doctor's recommendation". This information is useful but it is not considered critical. Notifications appear automatically at the right lower corner of the TV screen (see screenshot of Fig. 3a)). HDMI-CEC specifications limit the size of the message to only 13 characters. When the end-user perceives a notification, he/she presses the $\mathrm{OK}$ button of the remote command, and then the TV-set switches to HDMI2 showing the entire recommendation or warning. 

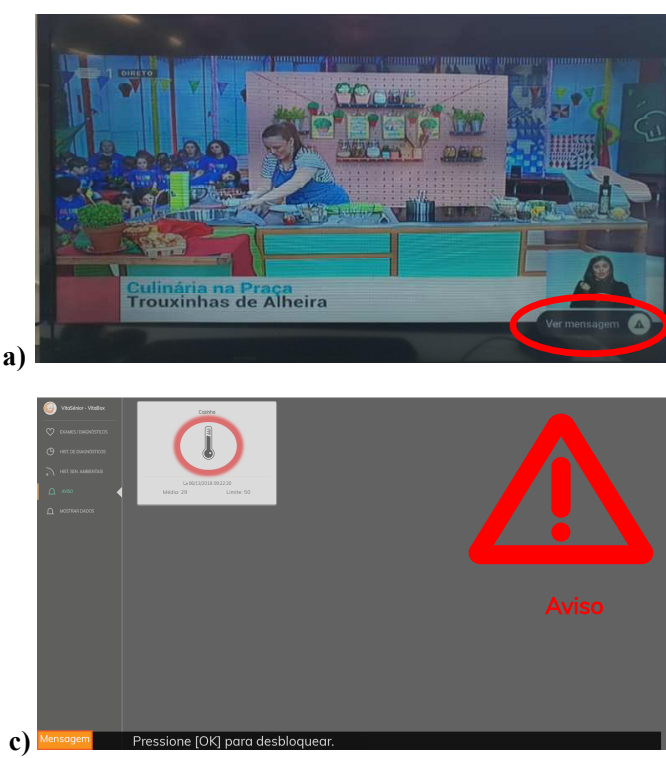
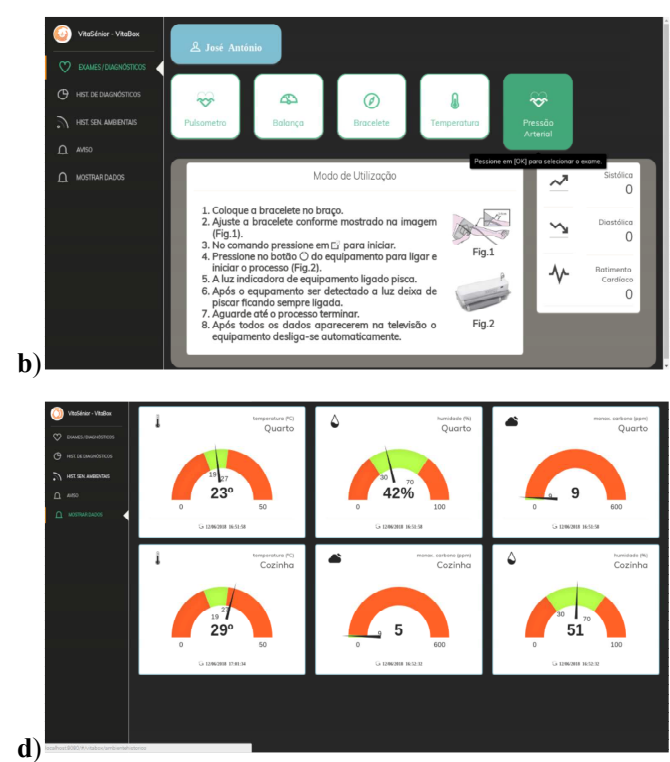

Fig. 3 Screenshots of some examples of user-TV interaction: a) Message notification blinking while end-user is watching TV; b) Recording of blood pressure and assistance procedure; c) Alert (automatic HDMI switching); d) Current environmental data visualization.

3) User interaction with medical devices for physiological measurement: The end-user measures the physiological parameters by his/her own initiative except for the wearable bracelet heartrate monitoring. Using the TV remote, the end-user selects the medical device he/she wishes to, by navigating the menu using the direction keys, the OK key and the Return key (6 keys) of the remote. A screenshot of the selection of the medical device is shown in Fig. 3b). After selecting the medical device, the TV shows the procedure to use the equipment and the user only needs to press OK (an example for blood pressure measurement is shown in Fig. 3b)). Depending of the medical device, the user may need to press a button on the device. When the measurement is finished, it is shown to the user, automatically recorded in the VITABOX and transmitted to the remote system in the cloud. The heartrate recorded from the wearable bracelet is automatically transmitted to VITABOX when the user is in its proximity, without any intervention of the user.

4) Data visualization and audio-description: The enduser can access and visualize at any moment the current environmental data and the latest records of biometric data. This information is shown with explicit dashboards (Fig. 3d)). Two modes were implemented to visualize the data, adjusted to end-user skills:

a) Basic mode: This mode minimizes the interaction of the end-user with the system. Simply pressing a key on the TV's remote control, the current environmental data and the latest biometric recordings are cyclically displayed accompanied by audio-description. Audio-description is also available for the interactions mentioned in 1) and 3). It is an important feature for end-users with vision impairments;

b) Advanced mode: This mode is targeted for more skilled end-users, who can efficiently navigate the menus. In this mode, users can access historical data recordings, but limited to the latest 10 biometric records, or the latest 24 hours of environmental parameters. To consult more data, users or caregivers need to access stored data from the remote server using a browser in a computer or mobile device.

\section{Software implementation}

VITABOX has a NodeJS server that makes available the whole interface for interaction, visualization of historical data and alerts. The MongoDB database is used to store the historical data and application settings required so that everything can work even if there is no Internet connection to the remote system. The server implements websockets technology that allows commands and data received from all interaction devices (TV remote control, WSN, and medical devices) interact with the interface. After local storage, data are sent to the remote system. The main software components are described below:

1) The Bluetooth interaction with the medical devices uses the Noble library [18] and associated dependencies installed on NodeJS. After the user selects the medical device, the interface informs the server of which Bluetooth device will be used. Then, the server searches the database for all the necessary information such that the whole process has the conditions to be executed. Then, the server constructs a child process exclusively to interact with the device. All relevant information is transmitted to the parent process to be stored and shown, and the child process is terminated.

2) To establish a communication channel between the border router of the WSN and NodeJS server, a SLIP tunnel was created and an UDP connection is opened using NodeJS datagram, managed by two child processes, one for the registration of the network sensors and requests' validation, and the other, a listener, which receives sensor data and alerts.

3) The text-to-speech conversion of the audio description module has been implemented without resorting to any of the various online services available. This ensures that audio-description is always available even if the Internet access is down, and reduces the overall data traffic of the 
proposed solution. Audio-description is based on a customized audio database of words and numbers. Each sentence of the audio-description results of the concatenation of the audio files into a single file. It uses the audioconcat library to access the ready-to-use methods provided by ffmpeg.

4) The overall user interface was developed in VueJS, with added modules such as vue-socket.io which uses websockets. It allows stateful communication between the VITABOX service and the client, making possible the remote-control interactions by the user. To promote a better user experience, an eventbus was integrated which permits the data flow in all the components of the application. Additional liraries were used to optimize the implementation. For example, the socket.io library uses the websockets to provide a bidirectional interaction with the browser, the nodecec library [19] uses the tools available from cec-utils to interact dynamically with hdmi-cec, and mongoose library is used to interact with mongodb.

\section{RESULTS AND CONCLUSIONS}

The software and hardware infrastructure of a complete IoT-based telehealth solution, VITASENIOR-MT, was implemented. VITABOX, the central component of the system, was set up in laboratory environment, simulating a real scenario, and tested by several people. Five medical devices with Bluetooth communication were tested to measure blood pressure, glucose, body weight, heart-rate and oximetry, and temperature, using the TV set for interaction. Environmental variables, including temperature, humidity, carbon monoxide and carbon dioxide, connected to a WSN, were continuously recorded for long periods of time. The sensors' transducers were validated with a commercial calibrated device, forcing abnormal conditions (for example, increasing gas concentration in test chambers). The IOC module is under development. The scalability of the system allows that new medical devices and environmental sensors can be easily added. The system manages all physiological and sensor data, storing them locally in the VITABOX and transferring them to the remote server, making them accessible via web browser to predefined users with different roles. This ensures that data are saved even when the Internet access is down. The functionalities of the TV interaction were tested regarding the use of medical devices and procedures, data visualization, alarm situations and recommendations.

The validation of this first telehealth prototype allows us to move to the second phase of the project, involving real endusers. The system will be set up on three houses inhabited by elderly people (aged 65 or more) in the Medio Tejo region. In a first period, the system will be adjusted using a user-centered design approach, considering feedback of the end-users, and then finally the health monitoring solution will be tested for a few months under real conditions. The acceptance and usability assessment of the overall system, and in particular of the TV set, will only be possible at the end of this phase, which will involve also the family, caregivers and physicians.

It is consensual that technology should be designed and adapted to meet the specific needs of target end-users. On the other hand, technology should also be adjusted to the reality of each country and region, for example, regarding telecommunication infrastructures and available services, and sociodemographic characteristics of the population.

Overall, the proposed architecture is a user-centered solution, where the end-user is directly involved in the loop of the healthcare system through the interaction with the TV set, without forgetting the other players, thereby promoting the interaction between users, healthcare institutions and providers of the technological solution.

\section{ACKNOWLEDGMENT}

This work has been financially supported by the IC\&DT project VITASENIOR-MT CENTRO-01-0145-FEDER023659 with FEDER funding through programs CENTRO2020 and FCT.

\section{REFERENCES}

[1] E. Comission, "Population ageing in Europe: facts, implications and policies Outcomes of EU-funded research," 2014.

[2] OECD, "Health at a Glance: Europe 2010," OECD Publishing http://dx.doi.org/10.1787/health_glance-2010-en, 2010.

[3] L. Normie, "Technology for Ageing in Place," IFA global ageing, vol. 7, pp. 45-53, 2011.

[4] M. Bujnowska-Fedak e U. Grata-Borkowska, "Use of telemedicine-based care for the aging and elderly: promises and pitfalls," Smart Homecare Technology and TeleHealth, vol. 3, p. 91-105, 2015.

[5] S. Majumder, E. Aghayi, M. Noferesti, H. Memarzadeh-Tehran, H. Mondal, Z. Pang e M. Deen, "Smart Homes for Elderly HealthcareRecent Advances and Research Challenges," Sensors, mdpi, vol. 17, pp. 132, 2017.

[6] E. Seto, K. J. Leonard, C. Masino, J. A. Cafazzo, J. Barnsley e H. J. Ross, "Attitudes of Heart Failure Patients and Health care Providers towards Mobile Phone-Based Remote Monitoring," Journal of Medical Internet Research, vol. 12(4), 2010.

[7] V. Ribeiro, A. Martins, A. Queirós, A. Silva e N. Pacheco, "Usability evaluation of a health care application based on IPTV," Conference on ENTERprise Information Systems, vol. 64, 2015.

[8] D. Schreurs, M. Mercuri, P. J. Soh e G. . Vandenbosch, "Wireless Health Monitoring: Design Challenges," em 11th Int. Conf. on Telec. in Modern Satellite, Cable and Broadcasting Services, 2013.

[9] A. P. Pablo G.-Sánchez and J. González and A. M. Mora, "Deploying intelligent e-health services in a mobile gateway," Expert Systems with Applications, vol. 40, p. 1231-1239, 2013.

[10] H. Mshali, T. Lemloumab, M. Moloney e D. Magoni, "A survey on health monitoring systems for health smart homes," International Journal of Industrial Ergonomics, vol. 66, pp. 26-56, 2018.

[11] Q. Lin, H. Ni e X. Zhou, "An OSGi-Based Heath Service Platform for Elderly People," IEEE 14th Int. Conf. on e-Health Networking, Applications and Services (Healthcom), pp. 10-13, 2012.

[12] P. García-Sánchez, J. González, A. M.Mora e A. Prieto, "Deploying intelligent e-health services in a mobile gateway," Expert Systems with Applications, Elsevier, vol. 40, p. 1231-1239, 2013.

[13] M. Pham, Y. Mengistu, H. Do e S. and Weihua, "Delivering home healthcare through a Cloud-based Smart Home Environment (CoSHE)," Future Generation Computer Systems, Elsevier, vol. 81, p. 129-140, 2018.

[14] M. Foster e K. Sethares, "Facilitators and Barriers to the Adoption of Telehealth in Older Adults: An Integrative Review," CIN: Computers, Informatics, Nursing, vol. 32(11), p. 523-533, 2014.

[15] S. Spinsante e E. Gambi, "Remote Health Monitoring by OSGi Technology and Digital TV Integration," IEEE Transactions on Consumer Electronics, vol. 58(4), pp. 1434-1441, 2012.

[16] "Independa," [Online]. Available: https://independa.com/. [Acedido em June 2018].

[17] "e-lio," [Online]. Available: https://e-lio.fr/e-liobox. [Acedido em June 2018].

[18] Noble. [Online]. Available: https://github.com/noble/noble. [Acedido em June 2018].

[19] node-cec. [Online]. Available: https://www.npmjs.com/package/node-cec. [Acedido em June 2018]. 